\title{
WHAT MAKES A GOOD FORECASTER?
}

\section{Forecasting the market potential for new drugs is becoming an increasingly important strategy in the survival and success of pharmaceutical and biotechnology companies.}

The world's leading pharmaceutical companies invest billions of dollars each year in research and development (R\&D), with little or no guarantee of a return on their investment. According to the Tufts Center for the Study of Drug Development, the average cost of developing a new drug is US $\$ 802$ million, a figure driven largely by the cost of clinical trials. Therefore, to fund the development of new therapies, large pharmaceutical companies need to maintain a $10 \%$ revenue growth rate - the equivalent of bringing two or three blockbuster drugs to market per year.

However, the current climate of depleted developmental pipelines and cost-containment policies by government agencies, as well as healthcare providers and payers, mean that pharmaceutical companies have a tough time ahead. Therefore, accurately forecasting the market potential for new compounds is becoming an essential tool in long-term strategic planning, as it aids in various decisions that are pivotal to the survival and success of a biotech or pharmaceutical company. Forecasting is used in many situations: to evaluate a licensing opportunity, to assess a particular lead compound and even in pipeline and R\&D portfolio analyses. Forecasting is also essential in understanding how the dynamics of a market are changing, and in raising awareness of a company's current and future competitors.

\section{What is involved in market forecasting?}

It is the forecaster's job to determine the current size of a particular market (called a base-year forecast) and then calculate how the dynamics of this market will change over the forecast period. (It is important to note that the largest markets are not necessarily the best to target with new compounds, as there may already be considerable competition, in which case the share that a new compound could expect will be small unless there are obvious clinical advantages.) To determine the base-year forecast, two approaches can be used. Top-down forecasting extrapolates from available sales data, using algorithms of how a particular drug class or market has previously performed. Bottom-up forecasting involves reconstructing the market from its components (BOX 1), which allows the analyst to model how particular changes over the forecast period will affect the base-year assumptions. A good forecaster is one who can reconcile both forecasting approaches.

\section{What experience is needed?}

Market forecasting within the pharmaceutical industry attracts people from a variety of backgrounds. A large proportion of forecasters have life-science qualifications (both graduate and postgraduate), with pharmacology and medi$\mathrm{cal}$ science being particularly useful when interpreting clinical data. Clinicians and pharmacists are also frequently employed. Pharmaceutical or biotechnology company experience is always useful: $\mathrm{R} \& \mathrm{D}$ scientists, clinical research associates and sales representatives can all provide valuable experience when trying to size a future market. However, not all market forecasters are science graduates. Social science is also well represented, as are business studies and marketing. What is essential is that the analyst understands the background to the area they are studying. Also, knowledge of current medical practice makes it easier to estimate what part future clinical changes might have on the market. Overall, an inquisitive mind and the ability to construct the big picture from small pieces of information are essential.

John Earl

\section{Box 1 | Successful bottom-up forecasting}

To create a successful bottom-up forecast, many variables of a given market need to be assessed.

Patient population. How is the population going to change over the forecast period? For example, degenerative conditions, such as Alzheimer's disease, and many cancers are increasing in incidence as the population ages. Will patient numbers increase because of new screening programs or improved diagnostics? Also, analysts have to factor for diagnosed patients that do not receive drug therapy - this omission could be because of cost or patient choice, or because drug therapy will be replaced by a medical device or surgical procedure.

Cost of therapy. A crucial component of all market forecasts is a consideration of what will happen to the price of drugs over the forecast period. Novel therapies that are more efficacious than existing therapies are typically priced at a premium. The analyst has to determine the number of patients switched to these newer, more expensive therapies and those who will still receive their existing medication. However, during the forecast, other drugs might lose their patent protection and subsequently be subject to generic competition. The level of generic competition varies between countries, but generics are almost always substantially cheaper than originator drugs. The analyst therefore has to determine the split of patients receiving the premium-priced branded agents versus those taking cheaper generic versions. The current trend for governments (especially in southern Europe) to promote increased use of generic drugs only complicates the picture for forecasters.

Other variables. Considerations that can also alter the cost of therapies are the dosing and duration of therapy. Clinical trial data might show that drug doses should be increased (which increases the market value) or decreased (which decreases the market value). The duration of a therapy may also be increased or decreased, which will have a positive or negative effect on the market, respectively. Patient compliance with a therapy also has an impact on the market potential of a new or existing therapy.

\section{(2) Online links}

\section{FURTHER INFORMATION}

The following associations are responsible for establishing standards and quality control in the pharmaceutical marketing research industry. European Pharmaceutical Market Research Association (EphMRA): http://umwephmro org/

The US-based Pharmaceutical Business Intelligence Group (PBIRG): http://www.pbirg.com/ Access to this interactive links box is free online
Resources Intl, 50-51

Russell Square, London

e-mail:

jearl@decisionresources.co.uk 